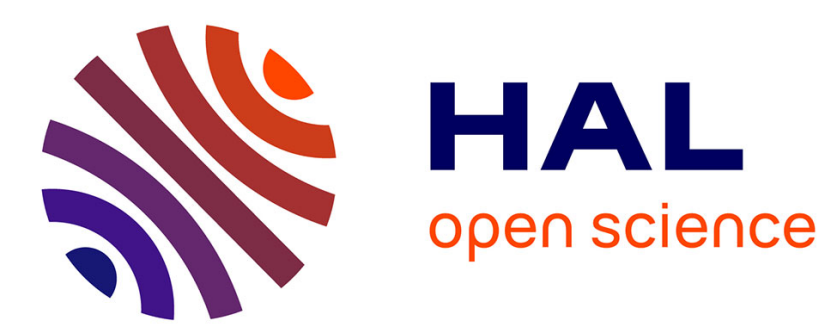

\title{
On the dynamics of quasi-steady gravity currents flowing up a slope
}

\author{
M.C. C de Falco, C. Adduce, M.E. Negretti, E.J. J Hopfinger
}

\section{To cite this version:}

M.C. C de Falco, C. Adduce, M.E. Negretti, E.J. J Hopfinger. On the dynamics of quasisteady gravity currents flowing up a slope. Advances in Water Resources, 2021, 147, pp.103791. 10.1016/j.advwatres.2020.103791 . hal-03001090

\section{HAL Id: hal-03001090 https://hal.science/hal-03001090}

Submitted on 23 Nov 2020

HAL is a multi-disciplinary open access archive for the deposit and dissemination of scientific research documents, whether they are published or not. The documents may come from teaching and research institutions in France or abroad, or from public or private research centers.
L'archive ouverte pluridisciplinaire HAL, est destinée au dépôt et à la diffusion de documents scientifiques de niveau recherche, publiés ou non, émanant des établissements d'enseignement et de recherche français ou étrangers, des laboratoires publics ou privés. 


\title{
On the dynamics of quasi-steady gravity currents flowing up a slope
}

\author{
M.C. De Falco, C.Adduce* \\ Department of Engineering, Roma Tre University, Rome, Italy \\ M.E. Negretti, E.J. Hopfinger \\ LEGI, UMR 5519, UGA/CNRS Grenoble France
}

\begin{abstract}
Quasi-steady gravity currents propagating first on a horizontal and then up a sloping boundary are investigated by means of theoretical analysis and laboratory experiments. The bottom slope ranged from 0.18 to 1 and full- and partial-depth configurations were considered. The developed theoretical model, using the depth averaged momentum equation, provides new physical insight into the importance of the different forces that act on the current and accounts for the gravity component along the slope, whose effect increases with both the slope angle and the ratio of current depth to ambient fluid. The height of the current decreases linearly with up-slope distance and the spatial rate of decrease, expressed by the current shape parameter is determined from the theory, using the measured up slope distance at which the current stops. This current shape parameter is found to depend on the slope only and it is not dependent on the current to ambient fluid depths. It can then be used to calculate the current velocity and the up-slope distance reached by the current. It is shown that the front velocity of all performed experiments is predicted by the theory indicating that the theory remains valid up to a slope equal to 1 .
\end{abstract}

Keywords: Gravity currents, theoretical model, experiments, sloping bottom

\section{Introduction}

Density-driven flows are ubiquitous in nature and examples are dense oceanic currents, sand storms or avalanches (Simpson, 1999). The dynamics of lock release gravity currents have been widely studied in the past, by means of laboratory experiments, high resolution numerical simulations and theoretical models on horizontal (Benjamin, 1968; Inghilesi et al., 2018; Kyrousi et al., 2018; Pelmard et al., 2018; Rottman and Simpson, 1983; Stancanelli et al., 2018a,b; Wilson et al., 2018, 2019; Zordan et al., 2018, 2019) and downsloping boundaries (Beghin et al., 1981; Dai, 2013a, 2014, 2015; Martin et al., 2019; Negretti et al., 2017; Ottolenghi et al., 2017b).

\footnotetext{
*Corresponding author

Email addresses: mariachiara.defalco@uniroma3.it (M.C. De Falco), claudia.adduce@uniroma3. it (C.Adduce), maria-eletta.negretti@legi.cnrs.fr ( M.E. Negretti), emil.hopfinger@legi.cnrs.fr (E.J. Hopfinger)
}

Preprint submitted to Advances in Water Resources 
Deterministic predictions of the speed of partial depth release gravity currents have been given by Benjamin (1968), and its analysis has been extended by Shin et al. (2004).

Only few studies have been conducted on gravity currents propagating up-slope (Cuthbertson et al., 2011; Jones et al., 2015; Laanearu et al., 2014; Ottolenghi et al., 2016, 2017a), although these flows occur frequently in nature. Salt wedges for example, are dense currents propagating up-slope along the river bottom and travelling upstream over long distances with implications on the coastal ecosystems. Avalanches have been observed to flow up a facing hill (Hopfinger, 1983). Internal solitary waves breaking at the continental shelf can develop gravity currents propagating up-slope (Helfrich, 1992; La Forgia et al., 2018a,b) affecting entrainment, mixing and sediment transport (La Forgia et al., 2020a,b). In stratified lakes and canyons, the geometric features of the sloping boundary, can affect the up-welling of dense deep waters induced by the strong impulses of wind (Cossu and Wells, 2013; Le Souëf and Allen, 2014; Shintani et al., 2010). Furthermore, sea breeze fronts can propagate inland and interact with an upsloping topography, affecting their dynamics and playing a key role on pollutants advection (Fernando, 2010). While the previous studies conducted on up-slope gravity currents focused on gravity currents propagating in a tilted channel with very small slope angles $\theta$, experiments on gravity currents propagating first on a horizontal bottom and then flowing up a slope $(0.25<S<1.15$, with $S=\tan \theta$ ) have been conducted by Marleau et al. (2014) using full and partial depth configurations for the initial lock conditions. They proposed a theoretical model using a WKB-like approach (Wentzel-Kramers-Brillouin) as Jones et al. (2015), who considered the front deceleration of a gravity current propagating up a V-shaped channel of small slope angle ( $S$ up to 0.14). In particular, Marleau et al. (2014) assumed that the front deceleration is caused only by the decreasing ambient fluid depth and neglected the effect of the along slope component of the gravity. Their experiments show that this assumption is reasonable when the ratio of current to ambient fluid depth is larger than 0.5 , indicating that for lower depth ratios, the effect of the gravity component along the slope cannot be neglected. Furthermore, a constant Froude number has been assumed while the current propagates up-slope in a decreasing ambient fluid depth $H_{s}(x)$, which is equivalent to hypothesize that the ratio $h / H_{s}$, with $h$ being the current depth, remains constant throughout the up-slope propagation. In a recent paper Zemach et al. (2019) used the Shallow Water (SW) model to simulate down-slope and up-slope gravity currents. This is a time dependent approach including also the dam-break process. De Falco et al. (2020) investigated the bulk entrainment in gravity currents flowing on horizontal boundary and then up a slope. It is shown that the contribution of the up-slope part of the current to bulk entrainment is negligible. In the present study, new experiments on lock-release gravity currents, in a configuration similar to Marleau et al. (2014) and De Falco et al. (2020), have been conducted with the current flowing first on a horizontal bottom and then up a slope where it comes to a stop. We expect the slow down of the current to depend on the gravitational term, and not only on the change in fluid depth. The theory developed here, is based on the depth averaged momentum equation (section 2) and is entirely different from that of Marleau et al. (2014). It provides new physical insight into the importance of the different forces acting on the current and accounts for the gravity component along the slope. This gravitational term is expected to be the main retarding force when the ratio of ambient fluid to current depth is large. In addition, the developed theory predicts the location $x_{F}^{\prime}$ at which the current stops, i.e. $U\left(x_{F}^{\prime}\right)=0$ provided the spatial variation of current height is known, and allows to calculate velocities along the slope. In section 4 we compare experimental results with the theoretical predictions, presented in section 2 . The experimental details are given in section 3 and concluding remarks in section 5. 


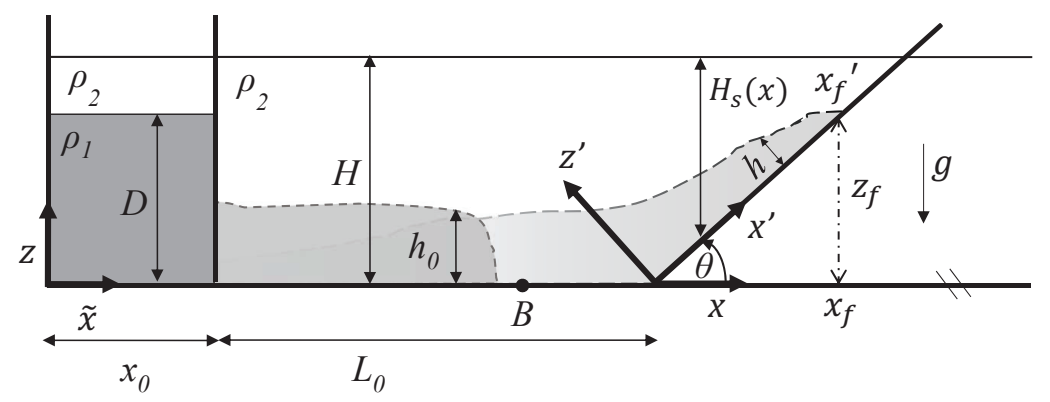

Figure 1: Sketch of the experimental apparatus and notations.

\section{Theoretical model}

The $(\tilde{x}, z)$ Cartesian coordinate system in Fig. 1 is oriented as the horizontal and vertical direction, while $\left(x^{\prime}, z^{\prime}\right)$ represents the coordinate system with respect to the inclined boundary, with origin at the toe of the slope. The coordinate $x$ is defined as $x=\tilde{x}-\left(x_{0}+L_{0}\right)$ and $x=0$ at the toe of the slope. The height of the current on the horizontal bottom is $h_{0}$, while on the inclined bottom is denoted by $h$ (Fig. 1). To be consistent with the coordinates used, the current height should be denoted $h^{\prime}$ to distinguish it from the vertical height. Since the vertical height is never used here, we drop the prime as is clear from Fig. 1. We start with the general governing $(\tilde{x}, z)$ equations using continuity and the Boussinesq approximation. Furthermore, making use of the boundary layer approximation $(w \ll u)$, the momentum equations reduce to:

$$
\frac{\partial u}{\partial t}+\frac{\partial u^{2}}{\partial \tilde{x}}+\frac{\partial u w}{\partial z}=-\frac{\partial}{\partial \tilde{x}}\left[g^{\prime}(h-z) \cos \theta\right]-g^{\prime} \sin \theta+\frac{\partial}{\partial z}\left(\frac{\tau}{\rho_{2}}\right)
$$

where $(u, w)$ are, respectively, the velocity components in the streamwise direction and perpendicular to the boundary, $\theta$ the slope angle (see Fig. 1), $\tau$ the shear stress, $g^{\prime}=g \Delta \rho / \rho$ is the reduced gravity, with $g$ the gravity acceleration and $\Delta \rho=\rho_{1}-\rho_{2}$, with $\rho_{2}$ the density of the ambient fluid and $\rho_{1}$ the density of the current.

For clarity the flow structure of the gravity current and the key phases of the interaction with the inclined bottom are shown in Fig. 2. When the gravity current reaches the inclined boundary, the dynamics of the current is strongly affected by the slope $S$, especially when $S \geq 0.58$. In general, while moving up the slope, the dense current decelerates due to changing ambient fluid depth and the along-slope gravity component, which acts against the motion (Fig. 2a), and comes to a stop at $x_{F}^{\prime}$ (time $t_{F}$ ). When $S<0.58$ the head of the current is clearly visible and the current thickness decreases linearly with up-slope distance up to $t=t_{F}$. Afterwards $\left(t>t_{F}\right)$ the current thickness decreases in time with an increase of the return flow causing an increase of current thickness at the beginning of the slope (Fig. 2b). The steeper is the slope, the larger is the part of the current that detaches from the main body travelling back and becoming part of the return flow (Fig. 2b). When $S \geq 0.58$, an increase at the toe of the slope is observed and it is caused by a reflection of part of the gravity current on the inclined boundary that acts like a barrier. We denote this process as splashing of dense current. In such cases the deceleration of the current is more rapid, the current thickness still decreases with up-slope distance, but an increase of the current thickness at the toe is found. In the following, the limit of the theory will be discussed 
with regard to $S \geq 0.58$.

The upsloping flow, until it comes to a stop, is predominantly spatially developing (Fig. 2a), i.e. the time-derivative term can be neglected with respect to the convective term. This assumption is supported by comparison of experiments with the theoretical results, notably the change in up-slope velocity, and by an evaluation of the time-derivative term with respect to the convective terms in Eq. (1). The time-derivative term is $\partial u / \partial t \sim \mathcal{U}^{2} / x^{\prime}{ }_{F}$, the time scale being $t \sim x_{F}^{\prime} / \mathcal{U}$ and the convective terms is $\partial u^{2} / \partial \tilde{x} \sim \mathcal{U}^{2} / \Delta x^{\prime}$, where $\mathcal{U}$ is a characteristic velocity. The ratio of time-derivative to convective terms is thus of order $\Delta x^{\prime} / x_{F}^{\prime}$, where $\Delta x^{\prime} \leq x_{f}^{\prime}$ is the distance over which the pressure changes. As indicated in Fig. 2, when the front comes to a stop, $x_{f}^{\prime} / x_{F}^{\prime} \rightarrow 1$ a temporal change in the current shape occurs. Neglecting the time-derivative term as long as $x_{f}^{\prime}<x_{F}^{\prime}$ implies that at position $x^{\prime}=x_{f}^{\prime}$ the velocity is constant or practically constant until the current comes to a stop. Experiments indicate that the change from spatial dependency of the flow to a time dependency is rather abrupt as sketched in Fig. 2. When the slope $S=0$, the ratio of time-derivative to convective terms is zero [velocity is constant provided the current is in the slumping phase, occurring up to $5.1 x_{0}$ when $\phi=0.3$ and $10 x_{0}$ from the gate when $\phi=1$ as in Rottman and Simpson (1983)] and it is small for moderate slope angles. On the contrary, when $S \gg 1$ convective terms become negligible. The limit of validity of the quasi-steady state assumption will be determined by comparison with experiments.

Eq. (1) can be integrated (Turner, 1973) over the full depth $(0-H)$ leading to

$$
\frac{d}{d \tilde{x}}\left[U_{2}^{2}(H-h)+U_{1}^{2} h\right]=-\frac{1}{2} \frac{d}{d \tilde{x}}\left(g^{\prime} h^{2} \cos \theta\right)-g^{\prime} h \sin \theta-\frac{\tau_{0}}{\rho_{2}} .
$$

where $U_{1}$ and $U_{2}$ denote the depth integrated velocities of the lower dense and upper fresh layers, respectively. From the volume flux conservation we have $U_{2}^{2}(H-h)=U_{1}^{2} h^{2} /(H-h)$, which can be used in Eq. (2) to give for the current velocity (dropping subscript 1)

$$
\frac{d}{d \tilde{x}}\left[U^{2} h H /(H-h)\right]=-\frac{1}{2} \frac{d}{d \tilde{x}}\left(g^{\prime} h^{2} \cos \theta\right)-g^{\prime} h \sin \theta-C_{D} U^{2} .
$$

where $C_{D}=\tau_{0} / \rho_{2} U^{2}$. As it is seen in Eq. (3) the main retarding mechanism is the back flow in the upper layer together with the along-slope gravity component. When $h / H \rightarrow 0(H \rightarrow \infty)$, the back flow contribution goes to 0 . The ratio of the bottom drag to the retarding gravity force is $C_{D} U^{2} /\left(g^{\prime} h \sin \theta\right) \sim C_{D} / \sin \theta$. The drag coefficient for the Reynolds numbers of the experiments ( $R e \gtrsim 3.3 \cdot 10^{3}$, see Table 1$)$, is $C_{D} \approx 10^{-3}$. Thus, the bottom drag is an order of magnitude less than the along slope gravity force, especially at larger slope angles; it can therefore be neglected. Following Rottman and Simpson (1983), dissipation effects are nevertheless included through a pre-factor $\beta$. It may be noted that if the gravity currents propagate in the form of a cloud, entrainment of ambient fluid into the current may become a major retarding mechanism as shown by Dai (2013b). Here for the current flowing up the slope, interfacial instabilities are suppressed and the entrainment can be considered negligible (De Falco et al., 2020).

On the horizontal boundary $\left(\tilde{x} \leq x_{0}+L_{0}\right), \theta=0$ and $d h / d \tilde{x}=d h_{0} / d \tilde{x}=0$ (when the bottom drag is neglected), thus Eq. (3) can be simplified to

$$
\left(U^{2} h\right) \frac{H}{H-h}=\left(U_{0}^{2} h_{0}\right) \frac{H}{H-h_{0}}=\text { const. }
$$

The constant (front condition) can be obtained from momentum conservation on a control volume including the head of the current that implies a balance between the pressure at the front of the 
current and ahead of it (location B in Fig. 1), $\Delta p / \rho_{2}=p_{0}-p_{B} / \rho_{2}$, i.e. the Froude condition of nose. For partial depth-release this gives (Rottman and Simpson, 1983):

$$
U_{0}^{2}=g^{\prime} h_{0}\left(1-\frac{\phi}{2}\right)\left(\frac{2-\frac{\phi}{2}}{1+\frac{\phi}{2}}\right)
$$

where $\phi=D / H$ is the depth ratio and $h_{0}=D / 2=\phi H / 2$ (Shin et al., 2004). Rottman and Simpson (1983) introduce a pre-factor $\beta^{2} / 2 \leq 1$ in Eq. (5), with $\beta^{2}$ ranging from about 1 to 1.6 , to account for viscous dissipation at the front and possible entrainment on the horizontal boundary, to adjust the front velocity to experiments.

On the inclined bottom $\left(\tilde{x} \rightarrow x^{\prime}\right.$ and $H \rightarrow H_{s}$ ), we can rewrite Eq. (3) as

$$
\frac{d}{d x^{\prime}}\left[U^{2} h H_{s} /\left(H_{S}-h\right)\right]=-\frac{1}{2} \frac{d}{d x^{\prime}}\left(g^{\prime} h^{2} \cos \theta\right)-g^{\prime} h \sin \theta,
$$

where $H_{s}$ is the total water depth above the inclined bottom boundary, decreasing linearly with $x$, i.e. $H_{s}=H-S x$, with $x=x^{\prime} \cos \theta$ (see Fig. 1). Integrating Eq. (6) with respect to $x$ from $x^{\prime}=0$ to $x^{\prime}$ gives:

$$
U^{2}=\left[\frac{1}{2}\left(g^{\prime} \frac{h_{0}^{2}-h^{2}}{h} \cos \theta\right)+\frac{g^{\prime}}{h} \sin \theta \int_{0}^{x^{\prime}} h d x^{\prime}-\frac{\beta^{2}}{2} \frac{g^{\prime} h_{0}^{2}}{h}\left(\frac{2-\frac{\phi}{2}}{1+\frac{\phi}{2}}\right)\right]\left(\frac{h}{H_{s}}-1\right)
$$

where for $U_{0}^{2}$, the Eq. (5) has been substituted, including a pre-factor $\beta^{2} / 2$ which will be determined from the experimental results.

To solve the remaining integral in Eq. (7), it is necessary to assume a dependence on $x^{\prime}$ of the current depth $h$. When the current propagates up the inclined bottom, the current thickness, which at the toe is constant and equal to $h_{0}$, is assumed to decreases linearly with up-slope distance (see Fig. 2) in the form:

$$
h=\left(h_{0}-C^{\prime} x^{\prime}\right)=\left(h_{0}-C x\right)
$$

where the current shape parameter $C=C^{\prime} / \cos \theta$. As previously discussed, return flow of dense current causes an increase of current height at the toe of the slope, which could cause a change in $h_{0}$ and determine a limit in the validity of $h_{0}=$ const. For small slope, the contribution to return flow is small, while since splashing is more pronounced, its contribution increases, for larger $S$, especially when $S \geq 0.58$. Nevertheless, for larger $S$, the deceleration of the front on the slope is more rapid, such that $x_{f}^{\prime} \rightarrow x_{F}^{\prime}$, when the reflection takes place (Fig.2), and the increase of $h_{0}$ at the toe of the slope becomes significant when $t>t_{F}$. The limit of this assumption will be discussed further in the results Sec.4 . Using Eq.(8), in Eq. (7) gives

$$
\begin{aligned}
& U^{2}=\left(\frac{h}{H_{s}}-1\right) \cdot\left(\frac{1}{h_{0}-C^{\prime} x^{\prime}}\right) \\
& {\left[\frac{1}{2} C^{\prime} x^{\prime} g^{\prime} \cos \theta\left(C^{\prime} x^{\prime}-2 h_{0}\right)+g^{\prime} x^{\prime} \sin \theta\left(h_{0}-\frac{C^{\prime} x^{\prime}}{2}\right)-\frac{\beta^{2}}{2} g^{\prime} h_{0}^{2}\left(\frac{2-\frac{\phi}{2}}{1+\frac{\phi}{2}}\right)\right] .}
\end{aligned}
$$

At the location $x^{\prime}=x_{F}^{\prime}$ the front velocity $U=0$ and we can determine $x_{F}^{\prime}$ from the following quadratic relation 


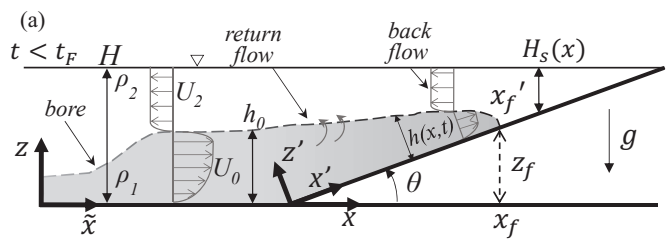

(c)
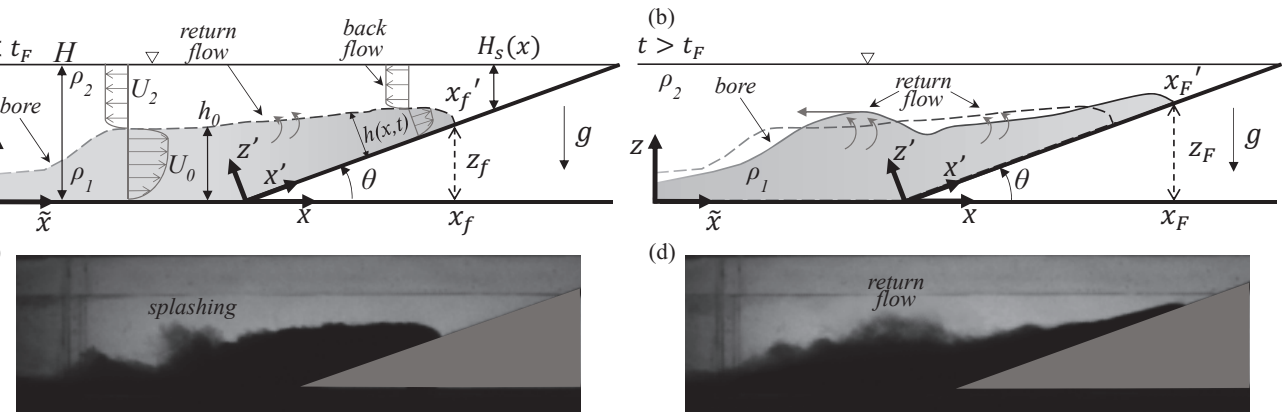

(d)

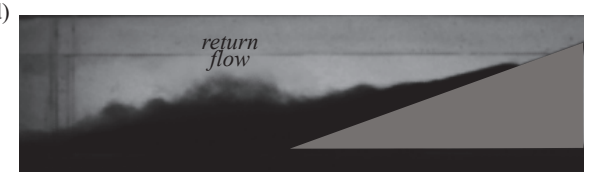

Figure 2: Schematic representation of the interaction with the inclined bottom and snapshots of the experiment with $S=0.36$ and $\phi=1$. (a) and (c) $t<t_{F}$ at time $t=7 \mathrm{~s}$, (b) and (d) $t>t_{F}$ at time $t=13 \mathrm{~s}$, where $t_{F}$ is the time at which the current front comes to a stop at $x_{f}^{\prime}=x_{F}^{\prime}$. The dashed line in (b) represents the interface of the dense current shown in (a).

$$
\frac{x_{F}^{\prime 2}}{2}\left(C^{\prime 2} \cos \theta-C^{\prime} \sin \theta\right)+x_{F}^{\prime}\left(h_{0} \sin \theta-C^{\prime} h_{0} \cos \theta\right)-\frac{\beta^{2}}{2} h_{0}^{2}\left(\frac{2-\frac{\phi}{2}}{1+\frac{\phi}{2}}\right)=0
$$

provided the constant $C^{\prime}$ is known. The assumption (8) gives

$$
\frac{h}{H_{S}}=\frac{h_{0}}{H} \frac{\left(1-\frac{C x}{h_{0}}\right)}{\left(1-\frac{S x}{H}\right)} .
$$

Marleau et al. (2014) assumed that the ratio of vertical current height to ambient fluid depth $h_{v} / H_{s}=h_{0} / H$ for all $\phi$. Since $h_{v} \approx h$ this implies, from Eq. (11), that $C \equiv C_{S} \approx S \phi / 2$. In section 4 a comparison between $C$ and $C_{S}$ is shown to determine whether $h_{v} / H_{s}$ is constant. Therefore the current shape parameter $C^{\prime}=-d h / d x^{\prime}$, is determined from measurements of $x_{F}^{\prime}$, using Eq. (10). As long as the assumption of stationary flow holds we can write $U=d x^{\prime} / d t$ and integrate Eq. (9), using $C^{\prime}$ to determine $x_{f}^{\prime}$. Comparing the solution with the experimental nose position gives an indication of the limits of validity of stationary flow assumption.

\section{Laboratory Experiments}

The laboratory experiments were performed at the Hydraulics Laboratory of Roma Tre University. A schematic representation of the tank used for each experimental run is shown in Fig. 1. The rectangular tank had a length $L=3.0 \mathrm{~m}$, width $W=0.2 \mathrm{~m}$ and depth $B_{d}=0.3 \mathrm{~m}$, with transparent Perspex walls and was uniformly back-lighted. The gravity current was produced by the lock-exchange technique applied to a saline mixture of initial density $\rho_{1}$. A vertical sliding gate was placed at the distance $x_{0}=0.2 \mathrm{~m}$ from the left side wall to separate the lock region of the dense salty water from the ambient freshwater of density $\rho_{2}$ in the tank. The reduced gravity $g^{\prime}$ was the same in all experimental runs, i.e. $g^{\prime}=0.3 \mathrm{~m} / \mathrm{s}^{2}$. The densities were measured by a density meter (Anton Paar DMA $4100 \mathrm{M}$ ), with an accuracy of $10^{-4} \mathrm{~g} / \mathrm{cm}^{3}$. A controlled quantity of dye was added to the lock salty water in order to visualize the flow and apply image analysis techniques. For each experiment, the tank was filled up to a total water depth of ambient fluid 


$\begin{array}{lcccc}\text { Label } & S=\tan (\theta) & \phi=D / H & h_{0}(\mathrm{~m}) & R e \\ 1 & 0.18 & 1 & 0.1 & 1.21 \cdot 10^{4} \\ 2 & 0.27 & 1 & 0.1 & 1.21 \cdot 10^{4} \\ 3 & 0.36 & 1 & 0.1 & 1.21 \cdot 10^{4} \\ 4 & 0.58 & 1 & 0.1 & 1.21 \cdot 10^{4} \\ 5 & 0.84 & 1 & 0.1 & 1.21 \cdot 10^{4} \\ 6 & 1.0 & 1 & 0.1 & 1.21 \cdot 10^{4} \\ 7 & 0.36 & 0.7 & 0.07 & 8.95 \cdot 10^{3} \\ 8 & 0.18 & 0.5 & 0.05 & 6.21 \cdot 10^{3} \\ 9 & 0.36 & 0.5 & 0.05 & 6.21 \cdot 10^{3} \\ 10 & 0.58 & 0.5 & 0.05 & 6.21 \cdot 10^{3} \\ 11 & 0.84 & 0.5 & 0.05 & 6.21 \cdot 10^{3} \\ 12 & 0.18 & 0.3 & 0.03 & 3.30 \cdot 10^{3} \\ 13 & 0.36 & 0.3 & 0.03 & 3.30 \cdot 10^{3}\end{array}$

Table 1: Main parameters varied in the experiments. $\phi=D / H ; R e=\rho h_{0} U_{0} / \mu ; H=0.2 \mathrm{~m} ; L_{0}=0.53 \mathrm{~m} ; g^{\prime}=0.3 \mathrm{~m} / \mathrm{s}^{2}$.

$H=0.2 \mathrm{~m}$. The depth-ratio $\phi=D / H$, with $D$ the depth of the salty water in the lock, was varied with $\phi=1, \phi=0.7, \phi=0.5$ and $\phi=0.3$, so that both, full and partial depth gravity currents were produced. A CCD camera, with a frequency of $25 \mathrm{~Hz}$ and space resolution of 1024 x 668 pixels, was used to acquire experimental images and the instantaneous density field $\rho(x, z, t)$ was evaluated by a light attenuation technique as in Nogueira et al. (2013). The dimensionless density field $\rho^{*}(x, z, t)$ is defined as:

$$
\rho^{*}(x, z, t)=\frac{\rho(x, z, t)-\rho_{2}}{\rho_{1}-\rho_{2}} .
$$

At the sudden removal of the gate, the dense water collapses under the freshwater and flows over the horizontal bottom before reaching the sloping boundary. The toe of the slope was placed at a distance $L_{0}=0.53 \mathrm{~m}$ from the vertical gate in order to have a gravity current propagating in the slumping phase. The slope angle $\theta$ ranged between $10^{\circ}$ and $45^{\circ}$, i.e. $0.18 \leq S \leq 1$. In Table 1 the main parameters varied in the experiments performed are summarized.

\section{Results}

\subsection{The normalized thickness}

In this section, a comparison between theoretical predictions of the current shape and experimental results obtained from the density field is presented. In particular, the assumption, made by Marleau et al. (2014), that $r_{h}=h_{v} / H_{s}(x)$ constant $\left(h_{v} \approx h\right)$, while the current develops, on both the horizontal and the sloping bottom, is herein discussed. Note that the analysis presented in Sec. 2, is based on the velocity thickness of the current over which the velocity is nearly uniform and has approximately the same speed as the front. At the outer edge the velocity decreases rapidly and reverses so that the velocity thickness threshold would correspond to about the $5 \%$ of the maximum dense current velocity. Differently, the density thickness of the current is defined by the interface between the dense and the light fluids, depending on the selected dimensionless density threshold. Since the velocity and density thicknesses are not identical we denote the density thickness by $h_{\rho}$. 
The space-time evolution of the normalized thickness $r_{h} / \phi=h_{\rho} /\left(\phi H_{s}\right)$ for the experiment with $\phi=1$ and $S=0.27$, for different dimensionless density thresholds, i.e. $2 \%, 20 \%, 50 \%$, used for the determination of the current thickness $h_{\rho}$, is presented in Fig. 3. Moreover, the integral thickness of Shin et al. (2004) is considered. The head of the gravity current is the raised region just behind the front, represented by the line which marks the transition from the white background to the color map area.

The black lines are the contours corresponding to different levels of $r_{h} / \phi$ while the vertical black line represents the toe of the slope. For a low threshold (i.e. 2\%, Fig. 3a), in the head region $r_{h} / \phi \simeq 0.5$, in agreement with the energy-conserving theory of Benjamin (1968), both on the horizontal and the sloping boundary. For higher thresholds, i.e. 20\% (Fig. 3b) and 50\% (Fig. $3 \mathrm{c}$ ), the normalized thickness $r_{h} / \phi$ of the head decreases and is about 0.4 and 0.3 , respectively. In Fig. 3d where $r_{h} / \phi$ is evaluated by considering the integral thickness of Shin et al. (2004), the current interface is lower and $r_{h} / \phi \simeq 0.3$ and is comparable to the $50 \%$ density threshold (Fig. $3 c)$. Therefore, when a high dimensionless density threshold is considered, the dense current is defined by a sharp interface between dense and light fluids and it does not include the mixing layer. This is also shown by subplots 1 and 2 corresponding to two different times in the spacetime evolution of $r_{h} / \phi$ marked by red dashed lines: (1) when the current reaches the toe of the up-slope and (2) when the current propagates on the inclined bottom. Different colors represent the threshold used for the definition of the interface of the dense current. As the threshold increases, the thickness decreases. The 50\% density threshold and the integral height of Shin et al. (2004), give similar values for the normalized thickness $r_{h} / \phi$.

The space-time evolution of $r_{h} / \phi$ is discussed for different $S$ and $\phi$ considering the $50 \%$ dimensionless density threshold for the definition of the interface of the dense current (Fig. 4).

For full-depth release experiments (i.e. $\phi=1$ ) in the head region $r_{h} / \phi \simeq 0.3$ during the whole propagation on the horizontal bed $(S=0$, Fig. 4a). In the up-slope experiments $(S=0.36$ in Fig. $4 \mathrm{~b}$ and $S=1$ in Fig. $4 \mathrm{c}), r_{h} / \phi \simeq 0.3$ on the horizontal part $(\tilde{x}<0.73)$ but an increase is observed near the toe of the slope, i.e. $\tilde{x} \simeq 0.73$. In particular for $S=1$ (Fig. 4c) the increase of $r_{h} / \phi$ at the toe of the slope is significant and is caused by a reflection of part of the gravity current on the inclined bottom boundary that acts like a barrier (see Fig. 2a).

For different depth-ratios (Fig. $4 \mathrm{~d}-\mathrm{f}$ ), $r_{h} / \phi \simeq 0.3 \div 0.4$ both on the horizontal and the sloping boundary, here for $S=0.36$. An increase of $r_{h} / \phi$ is observed right after the toe of the slope in the head region, subsequently the height of the current decreases with $x^{\prime}$, i.e. $\tilde{x}>0.73$. In particular, for $\phi=0.70$, in Fig.4d, the mean value of $r_{h} / \phi=0.41$ in the head region with a standard deviation $\sigma=0.15$ and a maximum $r_{h} / \phi_{\max }=0.7$ at $\tilde{x}=0.8 \mathrm{~m}$. For $\phi=0.5 \mathrm{in}$ Fig. $4 \mathrm{e}, r_{h} / \phi=0.41$ in the head region with a standard deviation $\sigma=0.12$ and $r_{h} / \phi_{\max }=0.73$ at $\tilde{x}=0.75 \mathrm{~m}$. Finally for $\phi=0.3 \mathrm{in} \mathrm{Fig.4f,}, r_{h} / \phi=0.46$ in the head region with a standard deviation $\sigma=0.12$ and $r_{h} / \phi_{\max }=0.75$ at $\tilde{x}=0.76 \mathrm{~m}$. Further, a comparison between the experimental front position, which is represented by the foremost point which marks the transition from the white background to the color-map area and the theoretical prediction is shown in Fig. 4. The red solid line represents the prediction of Eq. (5), on the horizontal bottom (i.e. on the left of the vertical black line which marks the toe of the slope) and Eq. (9) using $C^{\prime}$ on the inclined bottom (i.e. on the right of the vertical black line in Fig. 4). The latter has been integrated considering as initial position the beginning of the slope, and as initial time when the current starts to flow up the slope. For this reason a discontinuity in the red line occurs, close to the toe of the slope. The use of Eq. (5) when $\phi=1$ and $S=0$, reduces to the solution derived by Benjamin (1968), which is valid in the steady propagation of the front of the current, denoted $\tilde{x}_{f}$, up to $\tilde{x}_{f}(t) \sim 10 \tilde{x}_{0}\left(\tilde{x}_{f} \sim 2 \mathrm{~m}\right)$, when the self-similar regime starts (Fig. $\left.4 \mathrm{a}\right)$. The pre-factor 

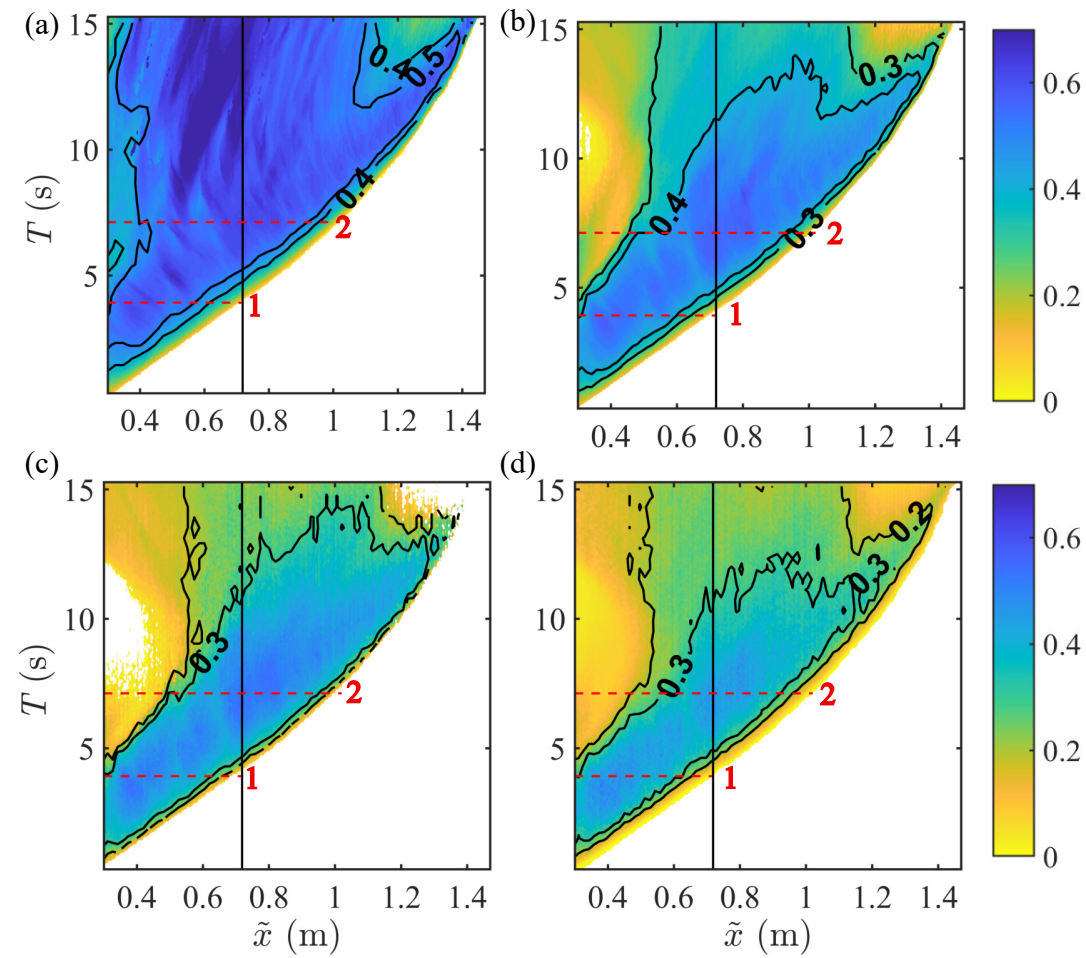

(d)
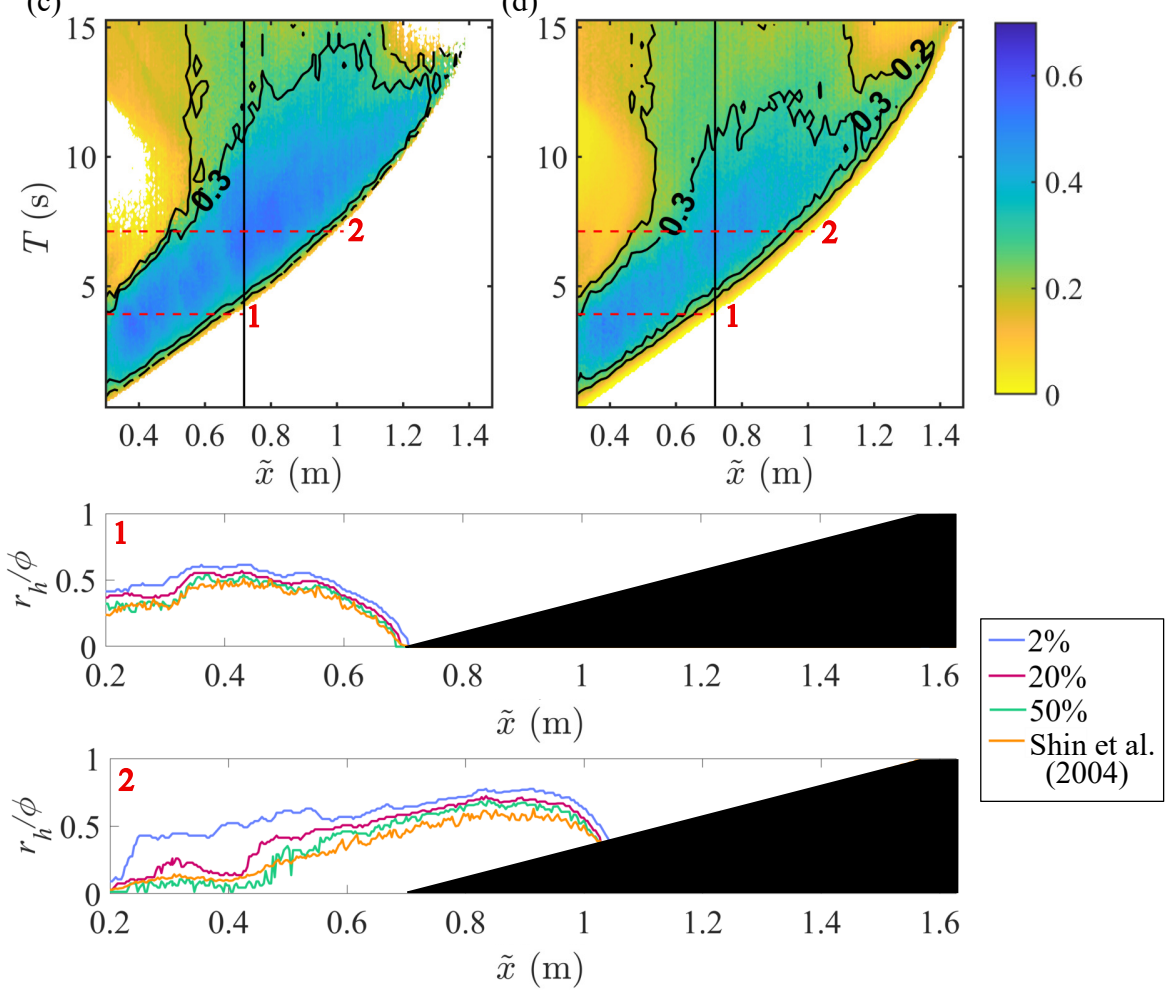

Figure 3: Space-time evolution of the normalized thickness $r_{h} / \phi$ for the experiment with $\phi=1$ and $S=0.27$ and for different thresholds: (a) $2 \%$, (b) 20\%,(c) $50 \%$, (d) the integral height of Shin et al. (2004). The vertical black solid line represents the toe of the slope while red dashed lines marks the time at which the normalized thickness with the four different density thresholds is evaluated: (1), when the current reaches the upslope and (2), when the current propagates on the upslope. 
(a)

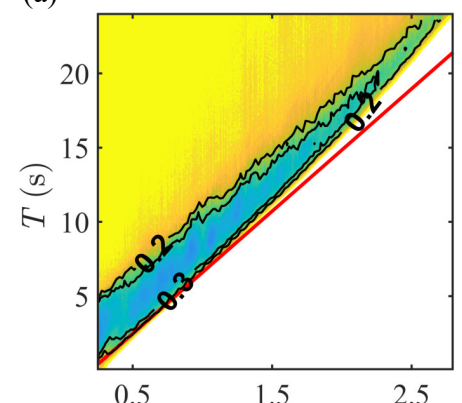

(b)

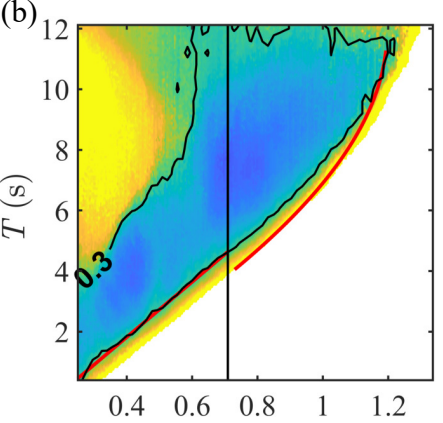

(c)

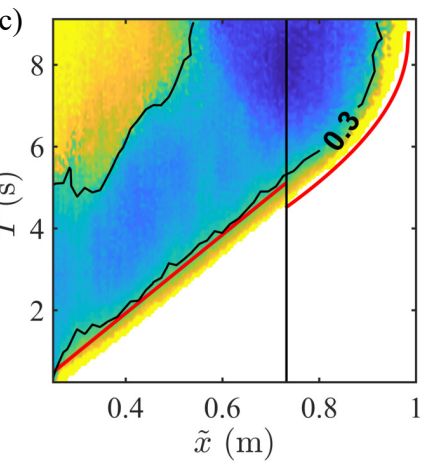

(d)

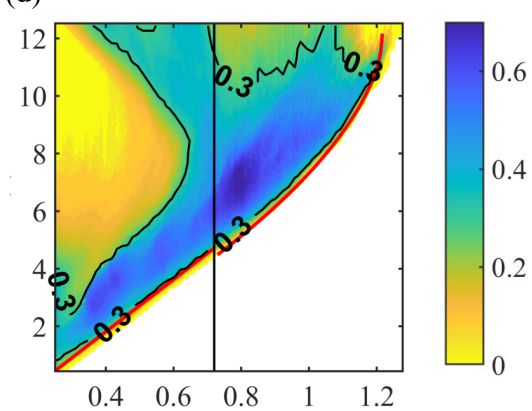

(e)

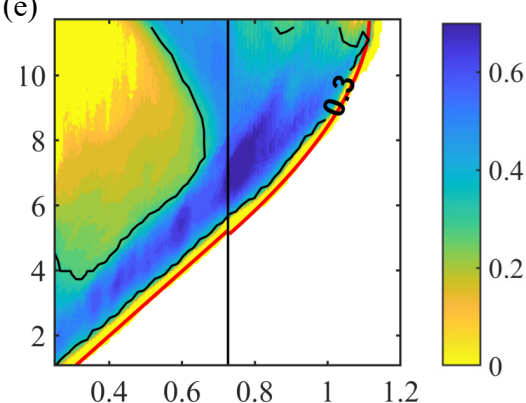

(f)

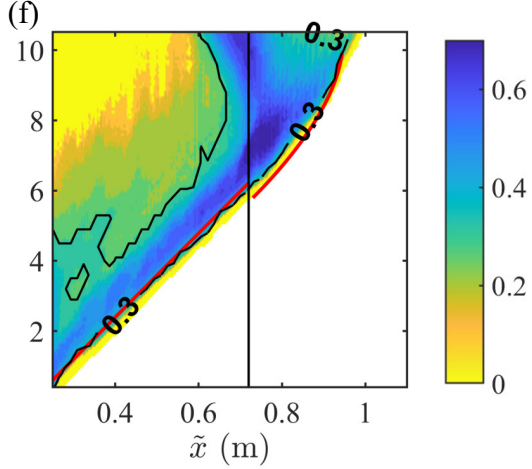

Figure 4: Space-time evolution of $r_{h} / \phi$ for $\phi=1$ (left panels) with (a) $S=0$, (b) $S=0.36$ and (c) $S=1$ and for $S=0.36$ (right panel) with (d) $\phi=0.7$, (e) $\phi=0.5$ and (f) $\phi=0.3$. The vertical black lines represent the toe of the slope and the red line represents the integration of Eq. (5), on the horizontal bottom and Eq. (9) on the inclined bottom using $C^{\prime}$ calculated from Eq. (10) using the measured $x_{F}^{\prime}$. 

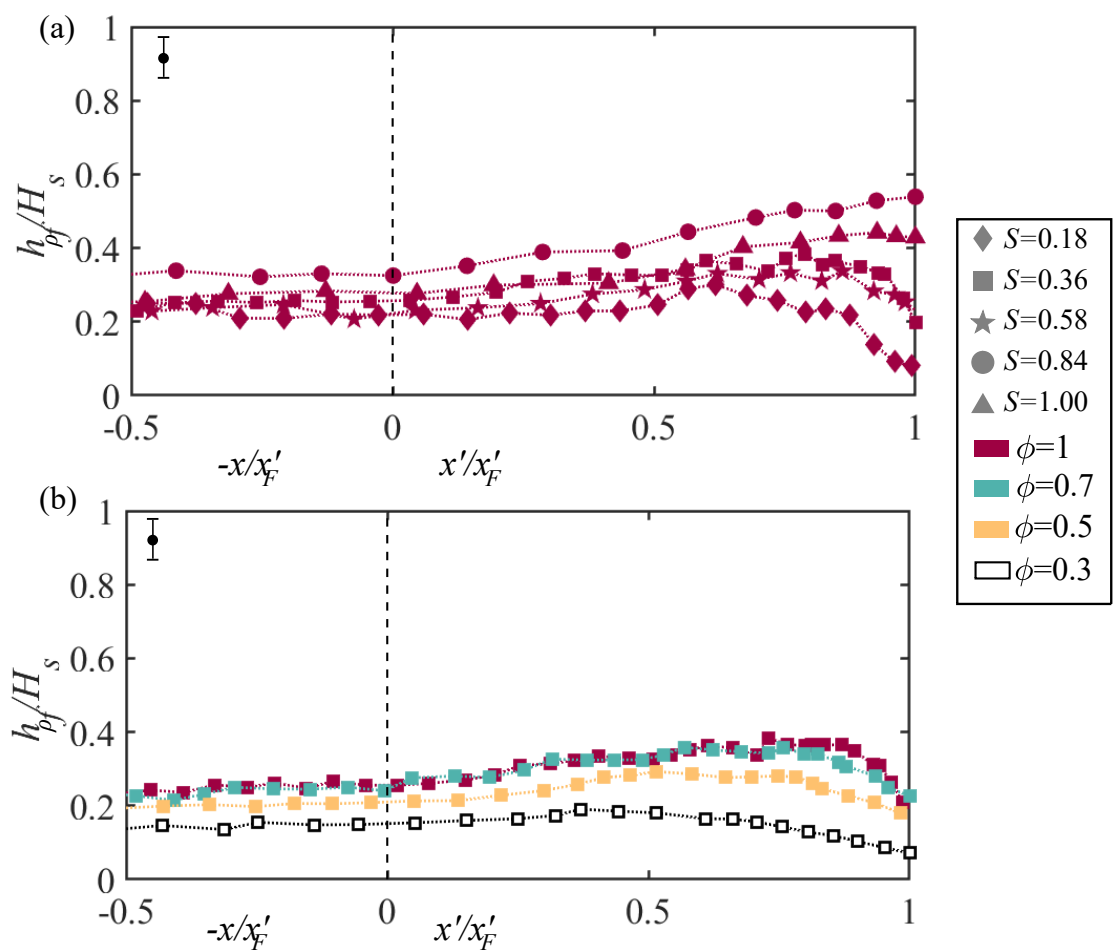

Figure 5: Dimensionless mean height $h_{\rho f} / H_{S}$ of the front vs. dimensionless front position $-x / x_{F}^{\prime}$ and $x^{\prime} / x_{F}^{\prime}$ : (a) $\phi=1$ and different $S$ ( $S=0.18, S=0.36, S=0.58, S=0.84, S=1)$; (b) $S=0.36$ and different $\phi(\phi=0.7,0.5,0.3)$. Dashed black line marks the toe of the up-slope.

$\beta^{2} / 2$ has been determined for each experiment, to adjust the front velocity to the experimental front. The pre-factor $\beta^{2} / 2$ ranges between 0.55 and 0.65 for $\phi=0.3$ and $\phi=0.5$, and lies between 0.7 and 0.8 for $\phi=0.7$ and $\phi=1$. For each $\phi$, the front position predicted by Eq. (5) on the horizontal boundary is consistent with the experimental results. On the inclined bottom, the predicted $x_{f}(t)$ in red is in good agreement with the experimental front position, when $\phi=1$ and $S=1$ (Fig. 4c) and when $S=0.36$ for $\phi=0.7$ and $\phi=0.5$ (Fig. 4d-e). When $S=0.36, \phi=1$ and $\phi=0.3$ the predicted final length $x_{F}^{\prime}$ is slightly lower than the one observed experimentally, but corresponds to the length reached by the bulk of the current (Fig. 4b,f). When the return flow increases, a decrease of $h$ in time (Fig. 2b) occurs and the current loses its typical shape. In this condition, the main part of the current comes to a stop, but a possible slight increase in the front position $x_{f}^{\prime}>x_{F}^{\prime}$ as the front thickness collapses to zero, can be observed.

The current thickness is also determined from the space-time density fields as a function of $x^{\prime}$, considering the mean thickness $h_{\rho f}$ of the current in the head region, $3 \mathrm{~cm}$ behind the nose of the current, determined by the $50 \%$ density threshold. Thus $h_{\rho f}$ is the density thickness, corresponding to $h_{0}$ on the horizontal and $h$ on the inclined bottom. In Fig. 5a, $h_{\rho f} / H_{s}$ is shown versus the non-dimensional front position $-x / x_{F}^{\prime}$ and $x^{\prime} / x_{F}^{\prime}$ for $\phi=1$ and different $S$ and in Fig. $5 \mathrm{~b}$ for $S=0.36$ and different $\phi$. The toe of the slope is represented by the dashed vertical black line. For $\phi=1, h_{\rho f} / H_{s} \simeq 0.3$ on the horizontal bottom, i.e. $x^{\prime}<0$, but it varies on the slope, 
i.e. $x^{\prime}>0$ (Fig. 5a) as a function of $S$. For fixed $S=0.36, h_{\rho f} / H_{s}$ varies with $\phi$ on both the horizontal bottom and the up-slope (Fig. 5b). The variation with $x^{\prime}$ of the current thickness $h_{\rho f}$ will be used in the following section, to compare the current shape parameter of the density field $-d h_{\rho f} / d x^{\prime}$ with $C^{\prime}$. It has to be noted that the definition of a certain region for the evaluation of the mean height of the current, adds a degree of uncertainty which is indicated by the error-bars in Fig. 5 so that the shape parameter obtained from the density fields is not very reliable.

\subsection{Final length and current shape parameter}

The dimensionless final height $z_{F} / h_{0}=x_{F}^{\prime} \sin \theta / h_{0}$ reached by the gravity current front is shown for each $\phi$ and $S$ investigated in Fig. 6a. Results are compared with the predictions of Marleau et al. (2014) (the slope ranged between $0.25<S<1.15$ ) who observed that the measured maximal height $z_{F}$ reached by the currents is constant varying $S$ and $z_{F} / D \simeq \gamma$, where $\gamma=0.86,0.99$ and 1.15 for respectively $\phi=1,0.75$ and $\phi=0.5$. The error-bars in these plots have been omitted because these are of size similar to the markers. The red line represents the height $z_{F}=D$, i.e. $z_{F} / h_{0}=2$. When $\phi<1, z_{F}$ is higher than the initial lock height $D, z_{F} / h_{0}$ increases with increasing $\phi$ and varies with $S$ especially for $\phi=0.5$.

The non-dimensional horizontal length $x_{F}$ reached by the current versus $S$ is shown in Fig. $6 \mathrm{~b}$ and it is compared with the predictions of Marleau et al. (2014) $\left(x_{F}=z_{F} / S=\gamma D / S\right)$ in black and a fairly good agreement is found. When $S$ is small, the final length reached by the dense current strongly depends on $\phi$, while as $S$ increases the influence of the depth ratio is lower and $x_{F}$ reaches a nearly constant value, not depending on $\phi$. Indeed, when $S \geq 0.58$, the current is more affected by the inclined bottom and the influence of $\phi$ becomes negligible. The positions $x_{F}^{\prime}$ measured in each experiment are used to calculate the current shape parameter $C^{\prime}=-d h / d x^{\prime}$, from Eq. (10). What emerges is that the current shape parameter $C=C^{\prime} / \cos \theta$, depends on $S$ and not on $\phi$ and increases as the slope angle increases. The best fit is $C \simeq 0.4 S$.

Fig. 6d displays the ratio $x_{F} C / h_{0}$ as a function of $S$ that appears in Eq. (8) when re-written in the form:

$$
\frac{h}{h_{0}}=1-\left(\frac{x_{F} C}{h_{0}}\right) \frac{x}{x_{F}}
$$

The factor $x_{F} C / h_{0}$ is representative of the self-similar shape of the current as considered by Marleau et al. (2014). When $x_{F} C / h_{0}=1$, the current height $h=0$ at $x=x_{F}$ and when $x_{F} C / h_{0}<$ 1 the current height is finite when the current comes to a stop at $x=x_{F}$. In some cases, as $S \leq 0.36$ and $\phi=0.3, x_{F} C / h_{0}$ is slightly larger than one, which means that the theoretical locations at which $h=0$ and $U=0$, appear for $x<x_{F}$, as seen in the velocity plots in Fig. $9 \mathrm{~b}$. Indeed, as observed in section 4.1 , when the return flow is observed, only a portion of the current continues to flow up-slope, while the bulk of the current stops (see Fig. 4f). As discussed by Marleau et al. (2014), on steep slopes the deceleration time of the current is short and no noticeable return flow occurs before the current reaches $x_{F}^{\prime}$. On the contrary, on small slopes the current takes a longer time to decelerate and return flow behind the front is possible, breaking the self-similar shape. However, even on the small slopes self-similarity is maintained to nearly $0.8 t_{F}$, where $t_{F}$ is the time when the front comes to a complete stop.

The current shape parameter has been also computed as the slope of the best fit of $h_{\rho f}\left(x^{\prime}\right)$, i.e. $-d h_{\rho f} / d x^{\prime}$, and it is compared in Fig. 7 with $C^{\prime}$, obtained from Eq. (10) (Fig. 6). There is good agreement between the shape parameter $C^{\prime}$ and $-d h_{\rho f} / d x^{\prime}$, when $S \leq 0.36$, whereas when $S>0.58, C^{\prime}$ is larger than $-d h_{\rho f} / d x^{\prime}$. The possible reason for the difference between $-d h_{\rho f} / d x^{\prime}$ and $C^{\prime}$ is that $-d h_{\rho f} / d x^{\prime}$ represents and is determined from the density field, while 

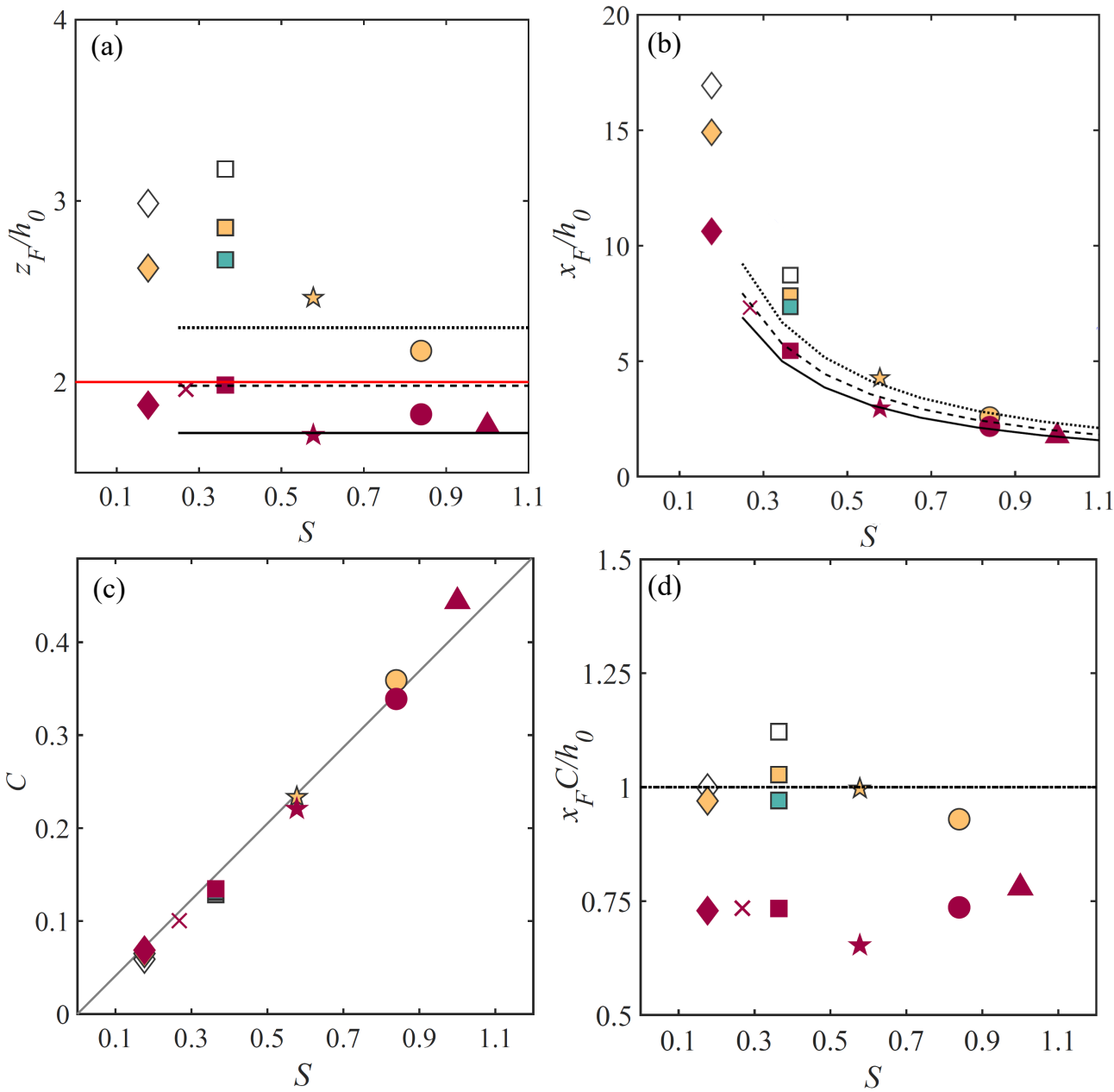

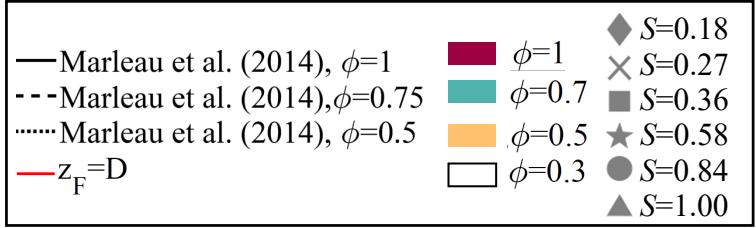

Figure 6: (a)Dimensionless final height $z_{F} / h_{0}$ versus slope $S$. The red line represents the height $z_{F}=D$; (b) $x_{F} / h_{0}$ vs $S$; (c) $C$ determined from Eq. (10) using the measured $x_{F}^{\prime}$ versus $S$. The slope of the gray solid line is 0.4 ; (d) the factor $x_{F} C / h_{0}$ vs $S$. 


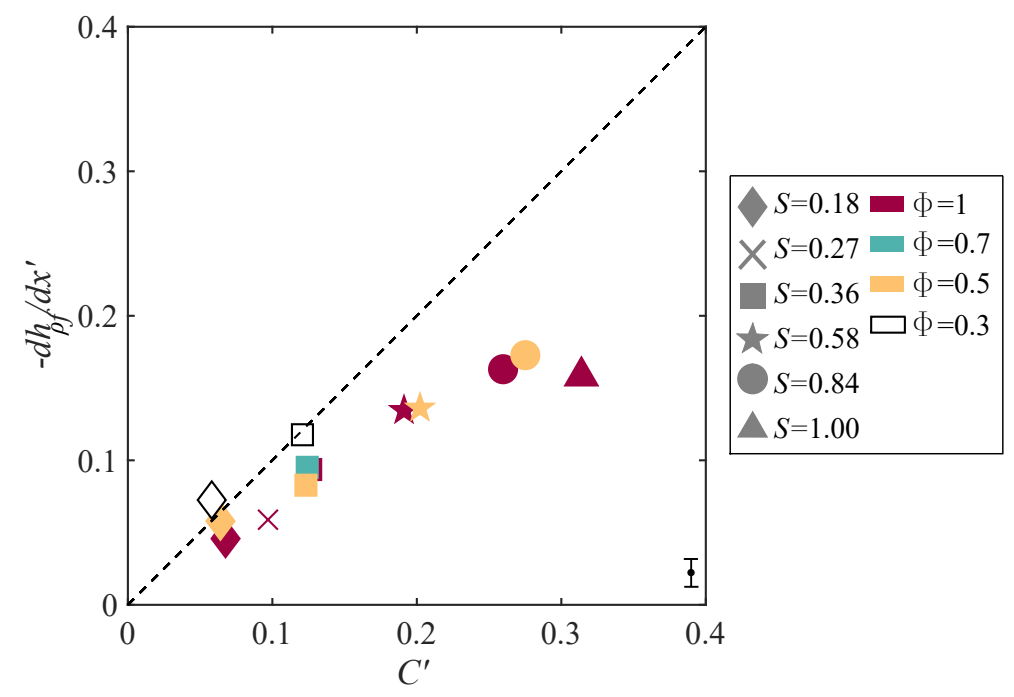

Figure 7: Comparison of $-d h_{\rho f} / d x^{\prime}$, obtained as the slope of the best fit line of $h_{\rho f}\left(x^{\prime}\right)$ with $C^{\prime}=C \cos \theta$ determined from Eq. (10) by considering the measured $x_{F}^{\prime}$.

$C^{\prime}$ is the change in velocity thickness, as previously discussed. Because of the return flow of dense current at the toe of the inclined bottom, the density interface slope $-d h_{\rho f} / d x^{\prime}$ is lower than the change in velocity thickness expressed by $C^{\prime}$. Since the return flow increases for larger slopes, when $S>0.58$ the difference increases. Moreover, for larger $S$, the definition of the head of the current on the inclined bottom is affected by the reflection and splashing, being more pronounced, which could further increase the difference. In addition, in such cases, due to return flow and reflection, the assumption $h_{0}$ is constant at the toe of the slope has limitation, which may also contribute to the discrepancy.

However, the important result is that the experiments confirmed that the current shape parameter has negligible dependency on $\phi$ and that $d h_{\rho f} / d x^{\prime}$ depends on the slope $S$ only. This is further confirmed by the ratio $h_{\rho f} / h_{0}$ that is shown in Fig. 8, together with the corresponding best fit lines determined for all data between $x^{\prime} / x_{F}^{\prime}=0$ and 1. In particular, for $\phi=1$ (Fig. 8a) and $\phi=0.5$ (Fig. 8c), the variation of $h_{\rho f} / h_{0}$ with $x^{\prime} / x_{F}^{\prime}$ and the slope of the best fit lines depend clearly on the slope $S$, while for a fixed $S$, the slope of the best fit lines weakly depends on $\phi$ and it is $\simeq 0.80-0.90$ for $S=0.36$ (Fig. 8b) and $\simeq 0.35$ for $S=0.84$ (Fig. 8d). This is in agreement with the behaviour of the shape parameter $C^{\prime}$, which, as shown in Fig. 6, depends on $S$ and and can be considered independent of $\phi$.

The coefficient of determination $R^{2}$ of the best-fit of $h_{\rho f} / h_{0}$, has been determined and it is found to be also dependent on $S$ and varies between 0.89 and 0.70 for $S<0.84$ and decreases below 0.70 for $S>0.84$. It is worth to note that low $R^{2}$ are found for larger $S$, which as shown in Fig.7 gives $-d h_{\rho f} / d x^{\prime}$, deviating from the values determined from equation (10) using measured $x_{F}{ }^{\prime}$. Two factors are expected to influence $R^{2}$. Because for larger $S$ the deceleration is more rapid and the distance of propagation on the inclined bottom is smaller, as shown in Fig.6b, such that a small number of data is available for the linear regression model and consequently a weak correlation is expected between the linear regression and $h_{\rho f} / h_{0}$. Moreover, low $R^{2}$ of the linear 

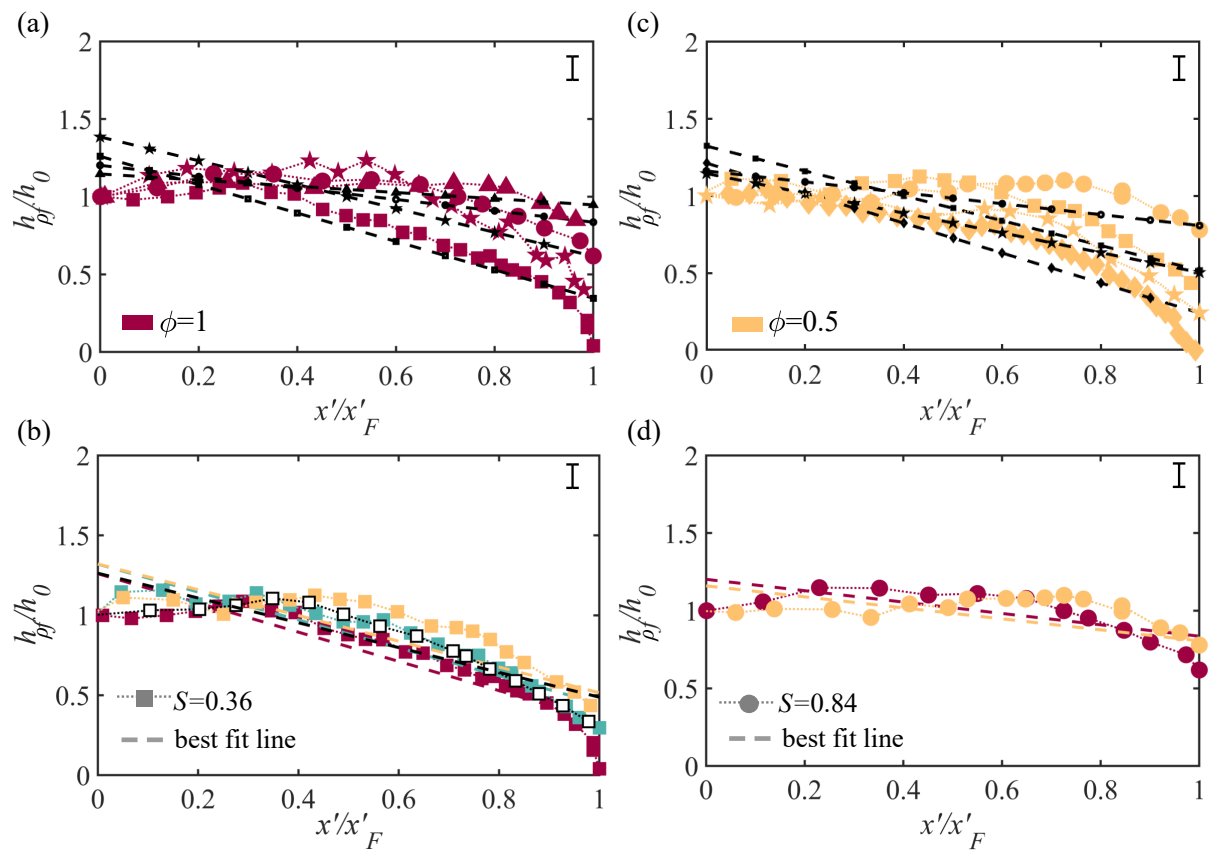

(d)

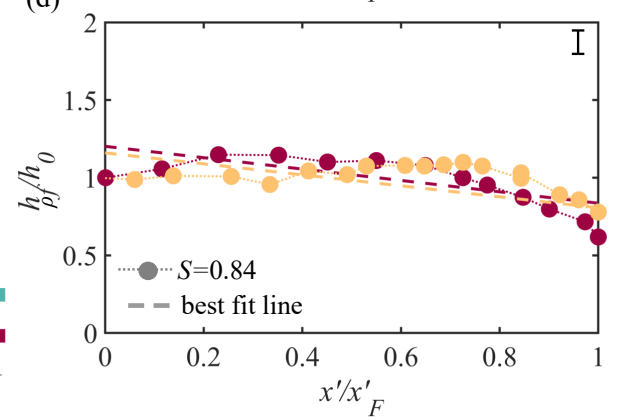

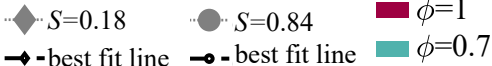

$$
\begin{aligned}
& \cdots=0.36 \quad-S=1.00 \quad \phi=0.5 \\
& \rightarrow \text {-best fit line } \rightarrow \text {-best fit line } \square \phi=0.3 \\
& \text { " } \pitchfork=0.58 \\
& \star \text {-best fit line }
\end{aligned}
$$

Figure 8: $h_{\rho f}\left(x^{\prime}\right) / h_{0}$ vs. $x^{\prime} / x_{F}^{\prime}$ : (a) $\phi=1$ and different $\theta(S=0.36, S=0.58, S=0.84, S=1)$; (b) $S=0.36$ and different $\phi(\phi=1,0.7,0.5,0.3)$;(c) $\phi=0.5$ and different $\theta(S=0.18, S=0.368, S=0.58, S=0.84)$; (d) $S=0.84$ and different $\phi(\phi=1,0.5)$

regression are also due to the difficulty in the clear definition of the dense current head for larger slopes, as previously discussed.

\subsection{Front velocity of the current flowing up the slope}

When the gravity current flows up the slope, it decelerates and stops when $x_{f}^{\prime}=x_{F}^{\prime}$. The experimental non-dimensional front velocity $U_{f} / U_{0}$ and $U / U_{0}$ predicted by Eq. (9), are plotted as a function of non-dimensional position $x^{\prime} / h_{0}$ (Fig. 9). By the assumption of steady current it is implicitly assumed that $U$ and $h$ at $x^{\prime}$ are equal to $U_{f}$ and $h_{f}$ at $x^{\prime}=x_{f}^{\prime}$, where $h_{f}$ is the front velocity thickness. The symbols in Fig. 9 represent the experimental non-dimensional front velocity, while the solid line is the mean velocity $U / U_{0}$, solution of Eq. (9), using $C^{\prime}$ obtained from Eq. (10) with the experimental values of $x_{F}^{\prime}$. The dimensionless velocities are compared for full-depth release experiments, $\phi=1$ by varying $S$ in Fig. 9a. As $S$ increases, the deceleration of the gravity current is larger and the run-up distance $x_{F}^{\prime}$ is lower. The effect of $\phi$ is shown for $S=0.36$ in Fig. 9b. It is seen that both, the experimental and the predicted dimensionless front velocity nearly overlap on one curve and are closely spaced for all $\phi$. This is supported by the 

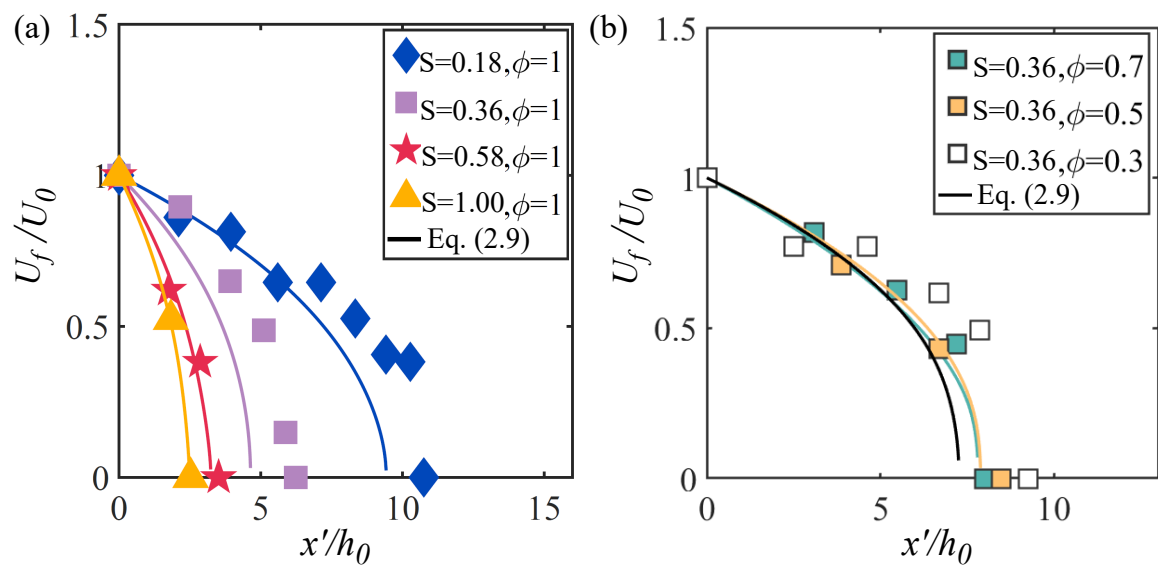

Figure 9: Dimensionless front velocity as a function of $x^{\prime} / h_{0}$. (a) $\phi=1$ varying $\theta(S=0.18, S=0.36, S=0.58, S=1)$ (b) $S=0.36$ varying $\phi(\phi=0.7,0.5,0.3)$.

observations (see Fig. $6 \mathrm{~b}$ of section 4.2), that the final length reached by the current depends on $S$ only, i.e. it is independent of $\phi$. There is fair to good agreement between the experimental dimensionless velocities and those predicted by Eq. (9) for all $S$ and $\phi$ investigated, especially at larger slopes when the time of deceleration is short. On shallower slopes deceleration times are longer so that return flow may increase (see Fig. 2b) before the current front comes to a stop. Nevertheless, as shown in Appendix A, at small angles, predicted velocities are also very sensitive to slight changes (error) in $C^{\prime}$.

Eq. (9) and (10) are valid for any $\phi$, including $\phi=0$, i.e. an infinite ambient fluid depth. For this case, a solution exists for $C^{\prime} \leq \frac{\sin \theta}{2 \beta^{2}+\cos \theta}$ which gives $x_{F}^{\prime} / h_{0} \leq 25$ for $S=0.18$.

\section{Conclusions}

The dynamics of quasi-steady gravity currents propagating up a slope has been investigated by a novel theoretical analysis and laboratory experiments. Full- and partial-depth lock release experiments were conducted by varying the current to ambient fluid depth-ratio proportional to $\phi$ and the slope $S$ from 0.18 up to 1 . The experiments focused on evaluating, from the density fields, the thickness of the dense current and the up-slope distance reached, using a light attenuation technique as well as on the change in up-slope front velocity.

The theory developed herein, using the depth averaged momentum equation, provides new physical insight into the importance of the different driving and retarding forces. It accounts for the gravity component along the slope, whose importance increases with slope angle, especially when the ambient fluid depth is very large, such that $\phi \rightarrow 0$. The space-time evolution of $r_{h}=h_{\rho} / H_{s}$ depends on the threshold of the dimensionless density field used to define the height of the dense current. As the threshold increases, $r_{h}$ in the head region decreases. An important result is that the decrease in height of the current up the slope, expressed by the shape parameter $C^{\prime}=-d h / d x^{\prime}$, that is determined from the theory, using the measured distance $x_{F}^{\prime}$ at which the current stops, depends on slope only. The best fit is $C^{\prime} \simeq 0.4 \sin \theta$ or $C \simeq 0.4 S$; the effect of $\phi$ is negligible. This behaviour is confirmed by the thickness variation obtained from the density 
fields noting however that the slope of density thickness $d h_{\rho f} / d x^{\prime}$ is lower than the slope of velocity thickness $d h / d x^{\prime}$ with the difference increasing with increasing $S$. However, the functional dependencies on $S$ and $\phi$ are the same. The front velocity is well predicted by the theory for all experiments conducted, indicating that the theory can be applied up to slopes $S \approx 1$ although, as shown by the density plots, some splashing occurs already for slopes $S \geq 0.58$. The theory also predicts well the velocities on shallow slopes, although part of the dense fluid behind the head begins to reverse direction before the current comes to a complete stop. Furthermore, Eq. (9) and (10) remain valid for any $\phi$, including $\phi=0$, i.e. an infinite ambient fluid depth often encountered in nature. For this case, a solution exists for $C=\frac{S}{2 \beta^{2}+\cos \theta}$ which is close to the value of $C$ given in Fig. $6 \mathrm{c}$ and gives $x_{F}^{\prime} / h_{0} \simeq 25$ for $S=0.18$ for example.

The final length $x_{F}^{\prime}$ was defined as the foremost point reached by the current on the slope. The experimental results show that $x_{F}^{\prime}$ depends on $\phi$ for smaller inclinations of the bottom, while when $S$ increases, $x_{F}^{\prime}$ reaches a constant value and does not depend on $\phi$ nor $S$. The measured $x_{F}^{\prime}$ corresponds to a final height $z_{F}=x_{F}^{\prime} \sin \theta$ reached by the gravity currents for each $S$ and $\phi$. The results show that $z_{F}$ is predominantly dependent on $\phi$. In particular when $\phi=1, z_{F} \simeq H$, while when $\phi<1, z_{F}>D$ for any $S$. The lower $\phi$ is, the larger is the non-dimensional ratio $z_{F} / h_{0}$. The non-dimensional ratio $x_{F} C / h_{0}$ was also considered as a parameter to define the shape of the current up-slope. When $\phi=1, x_{F} C / h_{0}<1$ and $h \neq 0$ when the current stops, whereas when $\phi<1, x_{F} C / h_{0} \simeq 1$ and the current height $h \simeq 0$ when the current stops at $x_{f}^{\prime}=x_{F}^{\prime}$.

In summary, the theory is able to capture the experimental results, confirming the validity of treating the gravity current development as a space dependent problem up to $S \approx 1$ and time $t \leq t_{F}$. The current shape parameter $C \simeq 0.4 S$ determined here allows to calculate the current velocity and the run-up distance for slopes $S<1$ and any $\phi$ provided the oncoming flow at the toe remains constant during the run-up time.

\section{Appendix A. Sensitivity of up-slope front velocity to $C^{\prime}=-d h / d x^{\prime}$}

The sensitivity of $U / U_{0}$, Eq. (9), on $C^{\prime}$ is shown in Appendix A.1 for the case of $S=0.36$. The solutions of Eq. (9) considering the shape parameter $C^{\prime}$, obtained from Eq. (10) using the experimental final length reached by the current on the upslope $x_{F}^{\prime}$, is displayed (solid lines) together with solutions of Eq. (9) considering $C_{S}^{\prime}=S \phi / 2$ from Eq. (11) that assumes $h / H_{s}$ constant and $h_{0}=H \phi / 2$ (dashed lines). Moreover the solutions of Eq. (9) are shown considering a decrease and an increase of $1 \%$ on $C^{\prime}$ (solid lines with circles and solid lines with crosses respectively). The comparison with the non-dimensional velocity evaluated by assuming $C_{S}^{\prime}$ reveals high dependence of Eq. (9) on the shape parameter $C^{\prime}$ since the predicted velocity from Eq. (9) with $C_{S}^{\prime}$ does not reproduce the experimental data and completely fails to predict $x_{F}^{\prime}$. More importantly, a decrease by $1 \%$ of the nominal value $C^{\prime}$ in Eq. (10), results in a decrease by $8 \%$ of the final length $x_{F}^{\prime}$ for the case with $\phi=0.7$ and $3 \%$ for $\phi=0.3$. An increase by $1 \%$ of $C^{\prime}$ in Eq. (10) causes: for $\phi=0.7$ the velocity to diverge, while for $\phi=0.3$ an increase of $13 \%$ on the final length $x_{F}^{\prime}$, which is close to the measured value of $x_{F}^{\prime}$. Finally, for $\phi=0.3$ an increase by $1.5 \%$ of $C^{\prime}$ in Eq. (10) causes the velocity to diverge.

\section{Acknowledgements}

This research was funded by the Italian Ministry of Education, University and Research (MIUR) through the Departments of Excellence 2018-2022 Program. 

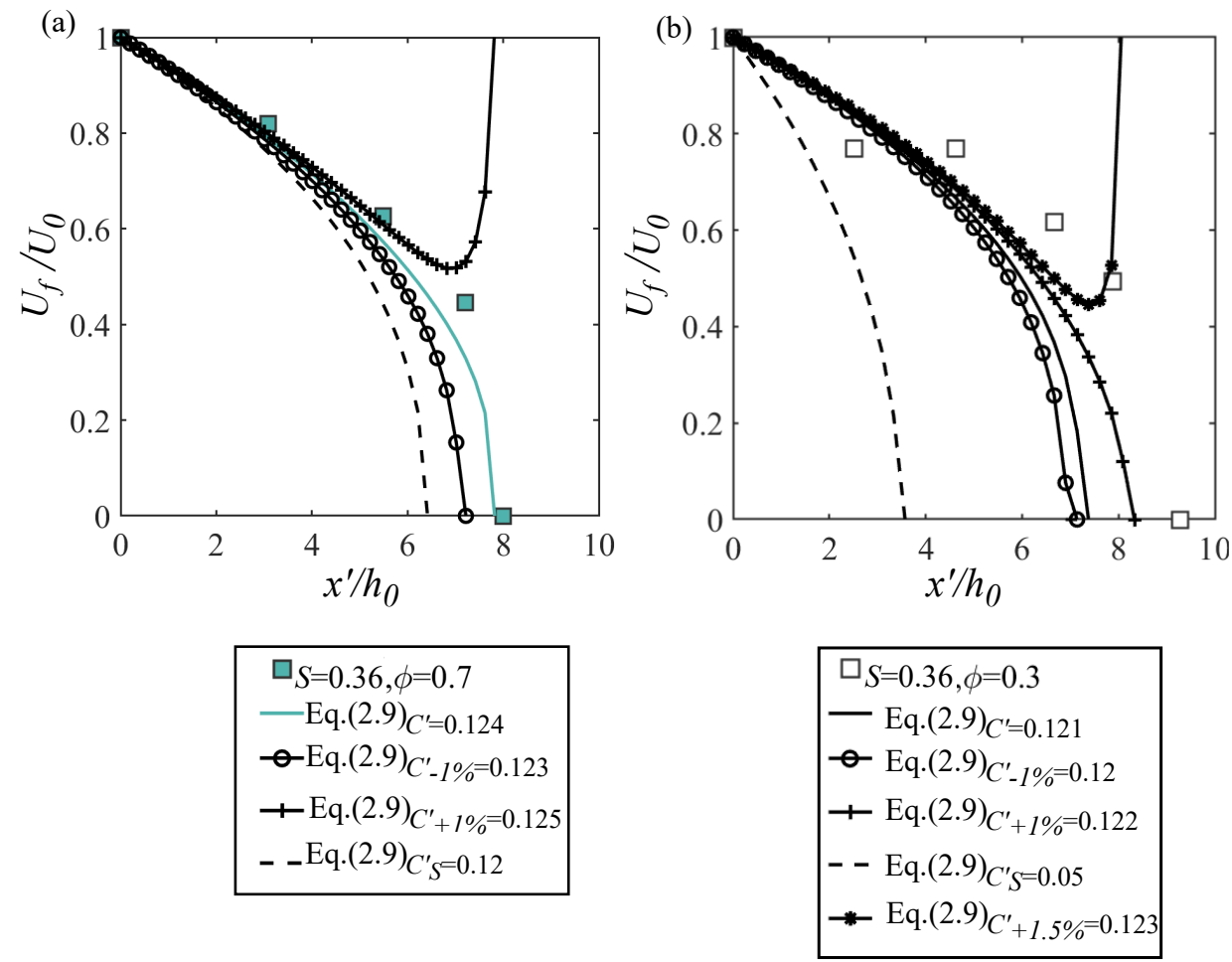

Figure Appendix A.1: Dimensionless front velocity versus $x^{\prime} / h_{0}$ for $S=0.36$ : (a) $\phi=0.7$ and (b) $\phi=0.3$. The squares represent the experimental instantaneous front velocity on the upslope, the solid lines represent the solution of Eq. 9 considering $C^{\prime}$, while the solid lines with circles and the solid lines with crosses represent the solutions of Eq. 9 considering a decrease and increase of $1 \%$, respectively, of the nominal value of $C^{\prime}$. 


\section{Declaration of Interests}

The authors report no conflict of interest.

\section{References}

Beghin, P., Hopfinger, E.J., Britter, R.E., 1981. Gravitational convection from instantaneous sources on inclined boundaries. J. Fluid. Mech. 107, 407-422.

Benjamin, T.B., 1968. Gravity currents and related phenomena. J. Fluid. Mech. 31.

Cossu, R., Wells, M.G., 2013. The interaction of large amplitude internal seiches with a shallow sloping lakebed: observations of benthic turbulence in Lake Simcoe, Ontario, Canada. PloS one 8, e57444.

Cuthbertson, A.J.S., Laanearu, J., Wåhlin, A.K., Davies, P.A., 2011. Experimental and analytical investigation of dense gravity currents in a rotating, up-sloping and converging channel. Dynam. Atmos. Oceans 52, 386-409.

Dai, A., 2013a. Experiments on gravity currents propagating on different bottom slopes. J. Fluid Mech. 731, 117-141.

Dai, A., 2013b. Power-law for gravity currents on slopes in the deceleration phase. Dynam. Atmos. Oceans 63, 94-102.

Dai, A., 2014. Non-Boussinesq gravity currents propagating on different bottom slopes. J. Fluid Mech. 741, 658-680.

Dai, A., 2015. High-resolution simulations of downslope gravity currents in the acceleration phase. Phys. Fluids 27, 076602.

De Falco, M.C., Ottolenghi, L., Adduce, C., 2020. Dynamics of gravity currents flowing up a slope and implications for entrainment. J. Hydraul. Eng 146, 04020011.

Fernando, H.J.S., 2010. Fluid dynamics of urban atmospheres in complex terrain. Annu. Rev. Fluid Mech. 42, $365-389$.

Helfrich, K.R., 1992. Internal solitary wave breaking and run-up on a uniform slope. J. Fluid Mech. 243, $133-154$.

Hopfinger, E.J., 1983. Snow avalanche motion and related phenomena. Annu. Rev. Fluid Mech. 15, 47-76.

Inghilesi, R., Adduce, C., Lombardi, V., Roman, F., Armenio, V., 2018. Axisymmetric three-dimensional gravity currents generated by lock exchange. J. Fluid Mech. 851, 507-544.

Jones, C.S., Cenedese, C., Chassignet, E.P., Linden, P.F., Sutherland, B.R., 2015. Gravity current propagation up a valley. J. Fluid Mech. 762, 417-434.

Kyrousi, F., Leonardi, A., Roman, F., Armenio, V., Zanello, F., Zordan, J., Juez, C., Falcomer, L., 2018. Large eddy simulations of sediment entrainment induced by a lock-exchange gravity current. Adv. Water Resour. 114, $102-118$.

La Forgia, G., Adduce, C., Falcini, F., 2018a. Laboratory investigation on internal solitary waves interacting with a uniform slope. Adv. Water Resour. 120, 4-18.

La Forgia, G., Ottolenghi, L., Adduce, C., Falcini, F., 2020a. Intrusions and solitons: Propagation and collision dynamics. Physics of Fluids 32, 076605.

La Forgia, G., Tokyay, T., Adduce, C., Constantinescu, G., 2018b. Numerical investigation of breaking internal solitary waves. Phys. Rev. Fluids 3, 104801.

La Forgia, G., Tokyay, T., Adduce, C., Constantinescu, G., 2020b. Bed shear stress and sediment entrainment potential for breaking of internal solitary waves. Adv. Water Resour. 135, 103475.

Laanearu, J., Cuthbertson, A.J.S., Davies, P.A., 2014. Dynamics of dense gravity currents and mixing in an up-sloping and converging vee-shaped channel. J. Hydraul. Res 52, 67-80.

Le Souëf, K.E., Allen, S.E., 2014. Physical modeling of tidal resonance in a submarine canyon. J. Geophys Res-Oceans 119, 1324-1343.

Marleau, L.J., Flynn, M.R., Sutherland, R., 2014. Gravity currents propagating up a slope. Phys. Fluids 26.

Martin, A., Negretti, M.E., Hopfinger, E.J., 2019. Development of gravity currents on slopes under different interfacial instability conditions. J. Fluid Mech. 880, 180-208.

Negretti, M.E., Flòr, J.B., Hopfinger, E.J., 2017. Development of gravity currents on rapidly changing slopes. J. Fluid Mech. 833, 70-97.

Nogueira, H.I.S., Adduce, C., Alves, E., Franca, M.J., 2013. Image analysis technique applied to lock-exchange gravity currents. Meas. Sci. Technol. 24, 047001.

Ottolenghi, L., Adduce, C., Inghilesi, R., Roman, F., Armenio, V., 2016. Mixing in lock-release gravity currents propagating up a slope. Phys. Fluids 28, 056604.

Ottolenghi, L., Adduce, C., Roman, F., Armenio, V., 2017a. Analysis of the flow in gravity currents propagating up a slope. Ocean Model. 115, 1-13.

Ottolenghi, L., Cenedese, C., Adduce, C., 2017b. Entrainment in a dense current flowing down a rough sloping bottom in a rotating fluid. J. of Phys. Oceanogr. 47, 485-498.

Pelmard, J., Norris, S., Friedrich, H., 2018. Les grid resolution requirements for the modelling of gravity currents. Computers \& Fluids 174, 256-270.

Rottman, J.W., Simpson, J.E., 1983. Gravity currents produced by instantaneous releases of a heavy fluid in a rectangular channel. J. Fluid. Mech. 135, 95-110. 
Shin, J.O., Dalziel, S.B., Linden, P.F., 2004. Gravity currents produced by lock exchange. J. Fluid. Mech. 521. Shintani, T., de la Fuente, A., de la Fuente, A., Niño, Y., Imberger, J., 2010. Generalizations of the Wedderburn number: Parameterizing upwelling in stratified lakes. Limnol. Oceanogr. 55, 1377-1389.

Simpson, J.E., 1999. Gravity currents: In the environment and the laboratory. Cambridge university press.

Stancanelli, L.M., Musumeci, R.E., Foti, E., 2018a. Computational fluid dynamics for modeling gravity currents in the presence of oscillatory ambient flow. Water 10, 635 .

Stancanelli, L.M., Musumeci, R.E., Foti, E., 2018b. Dynamics of gravity currents in the presence of surface waves. J. Geophys. Research: Oceans 123, 2254-2273.

Turner, J.S., 1973. Buoyancy effects in fluids. Cambridge university press.

Wilson, R.I., Friedrich, H., Stevens, C., 2018. Flow structure of unconfined turbidity currents interacting with an obstacle. Environ. Fluid Mech. 18, 1571-1594.

Wilson, R.I., Friedrich, H., Stevens, C., 2019. Quantifying propagation characteristics of unconfined turbidity currents interacting with an obstacle within the slumping regime. J. Hydraul. Res 57, 498-516.

down-slope or up-slope bottom. Phys. Fluids 31, 096604.

Zordan, J., Juez, C., Schleiss, A.J., Franca, M.J., 2018. Entrainment, transport and deposition of sediment by saline gravity currents. Adv. Water Resour. 115, 17-32.

Zordan, J., Schleiss, A., Franca, M.J., 2019. Potential erosion capacity of gravity currents created by changing initial conditions. Earth Surf. Dyns 7, 377-391. 